Revista Sostenibilidad, Tecnología y Humanismo

54

https://doi.org/10.25213/2216-1872.36

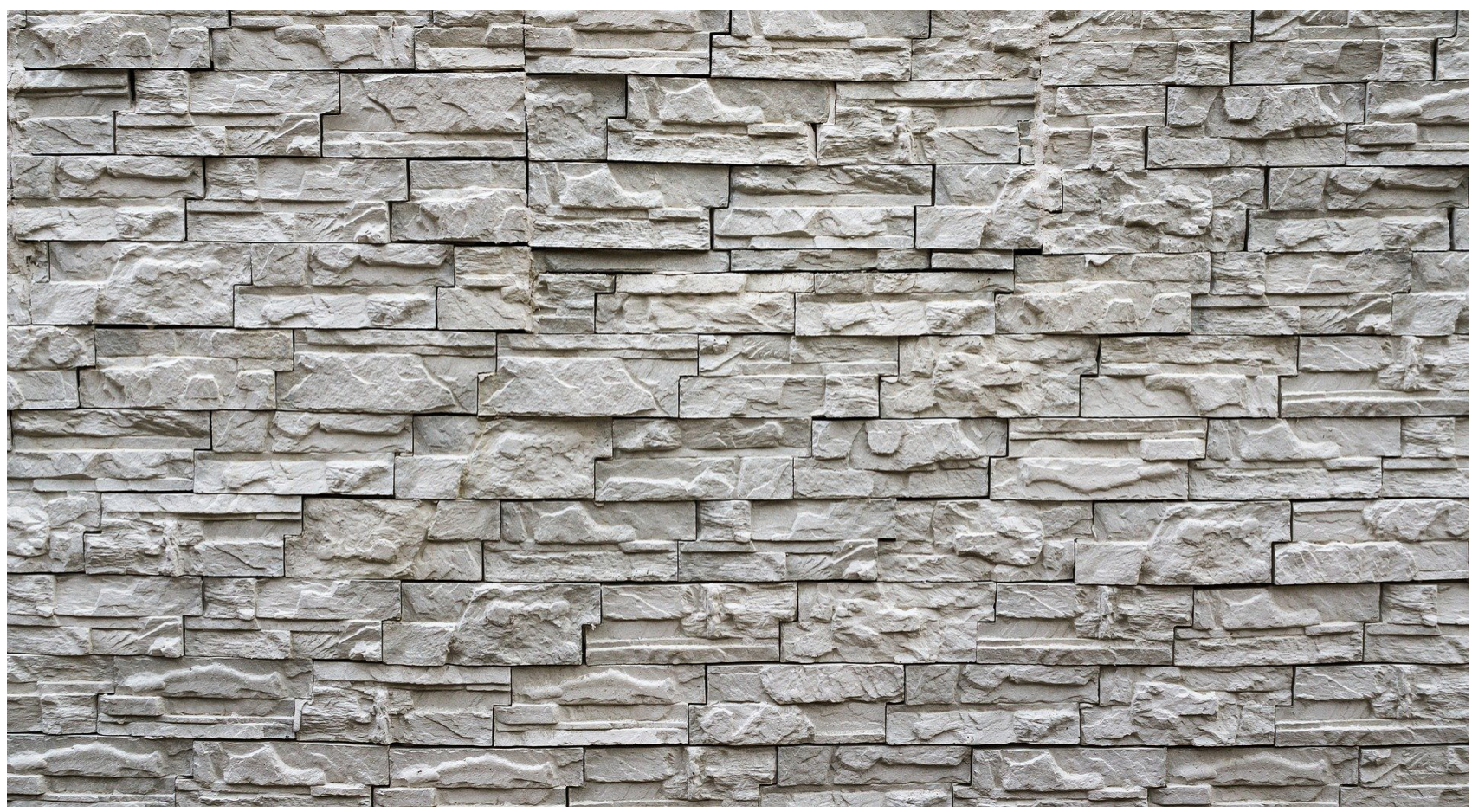

\title{
Análisis comparativo del desempeño de los ladrillos tradicionales frente a ladrillos pet
}

\section{Komparatif analysis kinerja batu traditional gown versus batu gown kesayangan}

Emmanuel Ortiz-Castellanos

Estudiantes de Ingeniería Civil, juanemmanueloc@ufps.edu.co, https://orcid.org/0000-0003-0866-3362,

Universidad Francisco de Paula Santander, Cúcuta, Colombia

Daniel Cristancho-Fernández

Estudiantes de Ingeniería Civil, danielalbertocf @ufps.edu.co,

https://orcid.org/0000-0003-4046-8938, Universidad Francisco de Paula

Santander, Cúcuta, Colombia

Brayan Avellaneda

Estudiante de Ingeniería Civil, brayanalbertoaf@ufps.edu.co,

Universidad Francisco de Paula Sanander, Cúcuta, Colombia. 
Resumen

El propósito de esta investigación fue analizar el desempeño de los ladrillos tradicionales frente a los pet, esto con el fin de establecer las diferencias que se puedan establecer con base a debilidades y fortalezas de cada uno frente a los diferentes factores establecidos para el análisis, encontró que contemplando la fabricación de ladrillos, el propósito de los ensayos de resistencia del concreto es la de determinar el cumplimiento de la especificación de resistencia y de medir su variabilidad, sin embargo estas variaciones también pueden ser el resultado de los procedimientos seguidos durante la dosificación, el mezclado, la colocación y el curado, además de estas, también se introducen variaciones en los ensayos por efecto de fabricación, por procedimiento de ensayo y por el tratamiento de las muestras de ensayo.

Palabras clave: Ladrillo, desempeño, características, cerámicos.

\section{Abstract}

The purpose of this research was to analyze the performance of traditional bricks against pet, this in order to establish the differences that can be established based on the weaknesses and strengths of each against the different factors established for the analysis, found that contemplating the manufacture of bricks, the purpose of the concrete resistance tests is to determine compliance with the resistance specification and to measure its variability, however these variations may also be the result of the procedures followed during the dosage, Mixing, placement and curing, in addition to these, variations are also introduced in tests by manufacturing effect, by test procedure and by treatment of test samples.

Keywords: Brick, performance, characteristics, ceramics. 


\section{6}

\section{Introducción}

Desde el principio de los tiempos el ser humano ha sido nómada y ha pasado de estar en constante movimiento y vivir en varios lugares, a ser sedentario y dejar esas costumbres en el olvido, evolucionaron hacia la cerámica y la escritura para tener una mejor calidad de vida, así fue como se empezó a pensar en las construcciones para comunicarse con las demás personas en residencias que tuviesen un periodo de vida extenso y permitieran a un gran número de personas convivir en armonía, de este mismo suceso surge la necesidad de aprovechar los recursos que brinda el entorno, gracias a esto el ser humano ha utilizado materiales como el cemento, concreto, hormigón, cerámicas, piedras entre otros.

Simultáneamente con el fin de encontrar una solución al problema del plástico en el medio ambiente, surge la idea de ver este material más como una solución que una problemática, dado que es "uno de los residuos más abundantes y no retornables denominado PET, que tarda en degradarse más de 500 años. Así que buscar un uso para su reciclado parece algo lógico" [1]. Aquí es donde inicia la caracterización de cada material para proceder a analizar las ventajas de cada uno frente al otro viendo como puntos principales la eficiencia, y generación de impactos ambientales y finalmente tener una comparación técnica, pero fácil de entender entre ambos materiales.

Primeramente, se explorarán los ladrillos convencionales que "son un elemento estable, durabley versátil, elaborado a partirde cerámica roja en combinación con otros elementos. Pueden presentarse en bloques sólidos y macizos, aireados, o con perforaciones que modifican sus características de resistencia, peso y uso [2]. Se fabrican mediante el proceso de cocción y debido a las características de las arcillas se aprovechan sus componentes fisicoquímicos, gracias al agua en su interior y su plasticidad es usado en todo el mundo; "sin embargo, aclara que la aplicación es económica si la construcción es eficiente, y en sistema aporticado la versatilidad que se logra en los espacios implica el uso del ladrillo" [3]. mayormente se usa para la construcción de muros, pero los estos tipos de ladrillos también se pueden usar para pavimentar carreteras de poco tránsito.

A pesar de las condiciones positivas presentadas por el ladrillo tradicional, en donde muestra varias facilidades de aplicación y beneficios económicos, también tiene sus desventajas e impactos negativos como: "ser los principales mecanismos de contaminación atmosférica por generar los procesos industriales que implican combustión que emiten gases contaminantes como el dióxido de carbono y monóxido de carbono, óxidos de nitrógeno, azufre, hidrocarburos, etc" [4]. Además se conoce que Colombia cuenta con un "depósito rico en arcilla apta para la elaboración de material cerámico" [5], pero la explotación de esta materia prima genera una grave desertificación del suelo.

Por otro lado, se tiene la problemática del plástico debido a que no es un material de fácil degradación, ya que reutilizarlo no es una opción pasada su vida útil. "Uno de los residuos más abundantes y no retornables que tenemos es el PET, que tarda en degradarse más de 500 años. Así que buscar un uso para su reciclado parece algo lógico" [5]. Se deben buscar nuevas alternativas para darle un nuevo uso mediante el reciclaje, y aquí es donde se da paso al siguiente planteamiento; Con la adición de plásticos reciclados, PET, BOPP, en morteros tradicionales se trata de obtener un material constructivo de características similares a los convencionales, brindando menor peso específico alcanzando resistencias exigidas por norma. "De esta manera coadyuvamos en el reciclado y la no contaminación por desechos plásticos." y así 
se puede tener un producto que combine las características del bloque tradicional con las ventajas del material plástico.

Para la preparación de este material constructivo se realiza el siguiente proceso que ayuda a combinar ambos materiales combinando sus propiedades, entonces "Los residuos se trituraran e incorporaron a una mezcla de cemento con un aditivo químico, para mejorar la resistencia, con esta mezcla se fabricaron ladrillos y tejas las cuales presentaron menor peso unitario, absorción aceptable y resistencias acordes a las exigidas por normas" [6] como resultado se obtiene un ladrillo más ecológico que otros tradicionales porque su materia prima principal está constituida por residuos plásticos reciclados.

Al producir este producto se busca competir con los productos existentes, teniendo esto en cuenta, se deben conseguir las mismas características o mejorar las propiedades y resultados al utilizarlo, conociendo que "pesan un kilo menos que un ladrillo convencional (ladrillo de PET pesa $1.400 \mathrm{~kg}$ )" [7]. Debatiendo sobre el material, se tiene que el plástico cuenta con ciertas características como la baja resistencia frente a la combustión pero "Ios ladrillos de PET con el cemento tienen buena resistencia al fuego, ya que los resultados del ensayo de propagación de la llama lo clasifican como material Clase RE 2: material combustible de muy baja propagación de llama" [6] con la aplicación de este ensayo no quedan dudas sobre la resistencia al fuego de los ladrillos PET.

En la adición de plásticos reciclados, en los productos de la construcción se tiene que es muy importante la cantidad de plástico agregado, según estudios adelantado por [8] "un ladrillo PET se fabrica reutilizando 20 botellas por lo que en ese aspecto cumple sobradamente", añadiendo otra característica positiva de los ladrillos PET es que son más livianos que los convencionales, un ladrillo convencional puede pesar en promedio $2 \mathrm{~kg}$ y un PET $1,4 \mathrm{~kg}$, aunque de manera individual no parezca mucha la diferencia, frente a un colectivo supone mucha reducción en cuenta a su carga estructural. "Y al utilizar una estructura más ligera también obtenemos edificios más económicos" [7]. En consecuencia, no solo pueden ser utilizados por ayudar el medio ambiente, sino que pueden afectar positivamente el diseño estructural de la edificación.

Añadiendo otra cualidad a este producto ecológico se tiene que "las paredes pueden ser diferentes entre sí; pero todas tienen algo en común: un coeficiente que mide su capacidad de aislación térmica llamado "k" (coeficiente de transmitancia térmica), cuanto menor es su valor, mayor la capacidad de aislación térmica que tendrá la pared" [9], y el ladrillo PET al tener esta ventaja tener un coeficiente de transmitancia térmica bajo nos da que "Entre las ventajas técnicas que nos puede aportar un ladrillo PET, la más interesante es su nivel de aislante térmico que es cinco veces mayor que la de un ladrillo tradicional. Este aumento sustancial del aislamiento puede llevar a reducir el grosor de los muros y pasar de cerramientos de 30 a $15 \mathrm{~cm}$ " [8], y el cambio de todas estas variables solo da mayores oportunidades de economía hacia un futuro, porque la climatización de la estructura será más optima lo cual lleva a utilizar en menos medida equipos de climatización.

Por otra parte, este tipo de ladrillo presenta una serie de beneficios ambientales, dado que el ladrillo se moldea con una máquina manual rodante y no necesita cocción en grandes hornos a cielo abierto (como sí lo requiere la fabricación del ladrillo de barro), "no produce contaminación atmosférica ni tala de árboles para obtener la leña necesaria para el funcionamiento del horno [10]. 


\section{8}

El uso de los ladrillos PET en Colombia no son una innovación pero en cuanto a investigación en el país no se ha profundizado. "El colombiano Fernando Llanos, haciendo uso de esta filosofía, logró encontrar en el plástico una solución de arquitectura, creando ladrillos con residuos de este material, que además tiene múltiples beneficios para el ámbito de la construcción" [11]. No solo tiene uso a un nivel arquitectónico, en cuanto a propiedades estructurales cumple con todos los requisitos que se exigen en la NSR-10.

El ladrillo PET no es la solución integral a la problemática ambiental generada por el exceso de plástico no reutilizable, pero es un buen inicio para contribuir a reducir su impacto, pero haciendo una comparación frente al ladrillo tradicional, el ladrillo PET tiene ciertas ventajas térmicas y lo iguala en cuanto a sus características de resistencia a tracción y compresión.

Esta investigación se realizó con el objetivo de comparar las características de eficiencia, viabilidad económica y generación de impactos ambientales de ambos materiales para su respectiva aplicación en la construcción, teniendo en cuenta sus usos y propósitos principales y con fundamento de la información hallada en distintas fuentes, las variadas reacciones en las formas de construcción teniendo como base los materiales de los productos utilizados.

Justificación

Esta discusión se realizó con el fin de identificar las posibles soluciones que se pueden entregar al momento de realizar una obra constructiva e implementar la construcción con ladrillos PET, las diferentes formas de construcción ya sean tradicionales 0 algunas alternativas que tienen efectos secundarios, "un ladrillo de PET se hace con 20 botellas descartables, lo que resalta la importancia ambiental de la investigación, ya que puede ser una alternativa al ladrillo de barro cocido que consume suelo fértil, usa leña de los bosques, y produce contaminación atmosférica" [12]. De esta manera se hace hincapié en como los ladrillos PET solo dan resultados positivos sin poner en riesgo el ambiente ni los recursos ambientales ni de ningún otro tipo.

\section{Bases teóricas}

Las investigaciones realizadas sobre los ladrillos PET tienen a CONICET (EI Consejo Nacional de Investigaciones Científicas y Técnicas) como sus pioneros en este campo, como ente autárquico dependiente del Ministerio de Ciencia, Tecnología e Innovación productiva de Argentina, señalando que "usamos PET procedente de envases descartables de bebidas y cemento Pórtland como ligante, más un aditivo químico que mejora la adherencia de las partículas plásticas al cemento" [13]. Los autores explican que la idea surgió de la cantidad de tiempo que estos materiales requieren para descomponer se y toman como base que es uno de los que más se acumulan, siendo la mejor alternativa con beneficios tanto sociales como ambientales.

Por otra parte, los ladrillos tradicionales que se usan en cualquier tipo de obra o vivienda destinada para uso de la comunidad, también se les denomina ladrillos no portantes a tubos horizontales, estos ladrillos cerámicos se utilizan para la fabricación de cualquier tipo de muro no portante, para cerramientos y fachadas, se utilizan para construcciones de muros a los cuales no se les transmiten cargas que provienen de todas las estructuras sismorresistentes como son vigas y columnas, se pueden elaborar para muros con diferentes espesores 10,15 o $20 \mathrm{~cm}$, por su poco peso, son la mejor opción para la construcción de tabiques sobre las placas de entrepiso, y son buenos aislantes térmicos. 
El plástico es un material que los podría sustituir ya que al utilizarlo en la construcción reduciría el impacto generado por utilizar materiales pétreos y así mismo ayudar con la reutilización del plástico que ha servido esencialmente al ser humano por su bajo costo de producción y porque suple el consumo masivo de materiales extraídos de los minerales terrestres. lo que implica la exigencia de mayor cantidad de recursos naturales tanto para alimentación como para obtener un techo digno y confortable. ¿De dónde se tendrá que extraer todos los recursos necesarios para construir esas viviendas? "El ritmo que es insostenible, no es el del crecimiento de la población mundial, sino el consumo que tenemos los habitantes y las empresas multinacionales del mundo occidental, que somos, realmente quienes estamos agotando los recursos del planeta" [14].

La producción de basura en las viviendas es un problema cultural y social muy difícil de solucionar, debido a la gran cantidad de desperdicios generados por unidad habitacional, más aún 17 el problema se agrava por la falta del proceso de reciclaje de estos 002C esto se evidencia a través del siguiente análisis: Según la Agencia Europea de Medio Ambiente dice, "En torno a una tercera parte de los recursos usados se convierten en residuos y emisiones. Cada año se generan cerca de cuatro toneladas de residuos per cápita en los países miembros de la AEMA. Cada ciudadano europeo se deshace de una media de $520 \mathrm{~kg}$ de residuos domésticos al año, y se espera que la cifra aumente" [14].

Materiales y métodos

Se realizó con base en una investigación descriptiva en donde la recolección de datos a través de distintas fuentes bibliográficas (libros y páginas web) y exponer los temas referentes al ladrillo PET como material de construcción. Asimismo el diseño de investigación documental, es el proceso de búsqueda de datos a través de páginas web, artículos, ensayos; sobre los ladrillos PET, sus características, ventajas y desventajas, que tan frecuente es el uso de este material en la construcción que requiera el uso de este material aislante y ecológico, para analizar esta información e incentivar a la investigación de procesos que hagan más efectivo y concurrido el uso del producto como principal material de construcción.

Variables

La utilización del ladrillo PET como material de construcción.

Definición de la variable: la importancia de los ladrillos PET en las construcciones, las ventajas y desventajas que trae consigo, la frecuencia con que es utilizado, la cantidad de edificaciones que han sido construidas a base a este, la tendencia de este material en Colombia, que regiones de Colombia es más utilizado el ladrillo PET como principal material de construcción, el concepto del presente ante la sociedad, y la implementación de nuevos proyectos que requieran su uso.

Técnicas e instrumentos de recolección de datos de análisis documental: recopilación de los distintos documentos que tratan los ladrillos PET, para analizarlos y dar posibles soluciones a la problemática ambiental, recursos financieros, obtención de materiales, tiempo y costos de obra, entre otros. 


\section{Primer análisis}

\begin{tabular}{|c|c|c|}
\hline Fuente & Descripción & Análisis \\
\hline $\begin{array}{l}\text { "LADRILLOS PEI" A BASE DE } \\
\text { RESIDUOS PLASTICOS CIENCIA } \\
\text { Y CFMENTO. (2015)."|16|. }\end{array}$ & 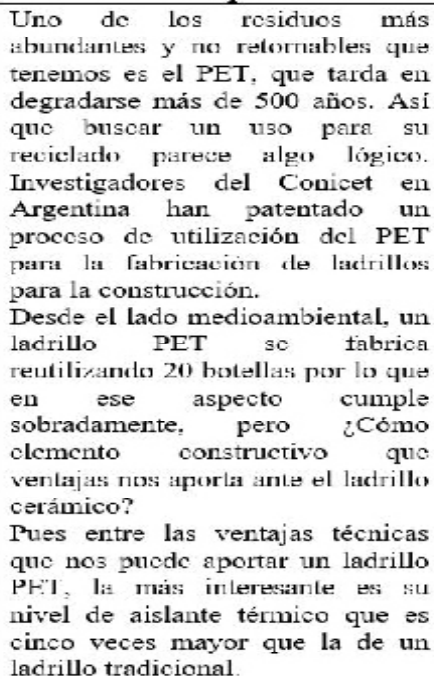 & 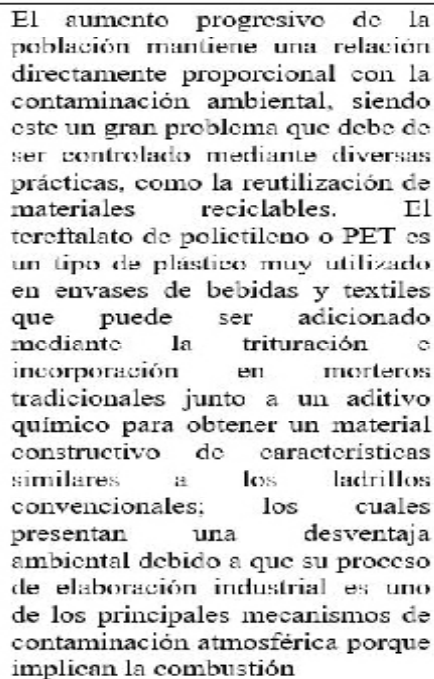 \\
\hline
\end{tabular}

\section{Segundo análisis}

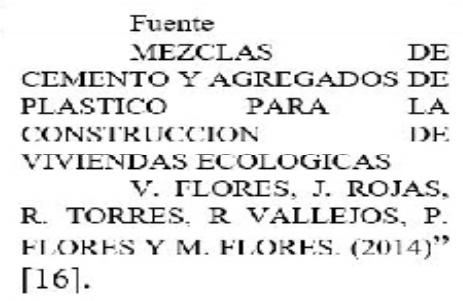
「16ך.

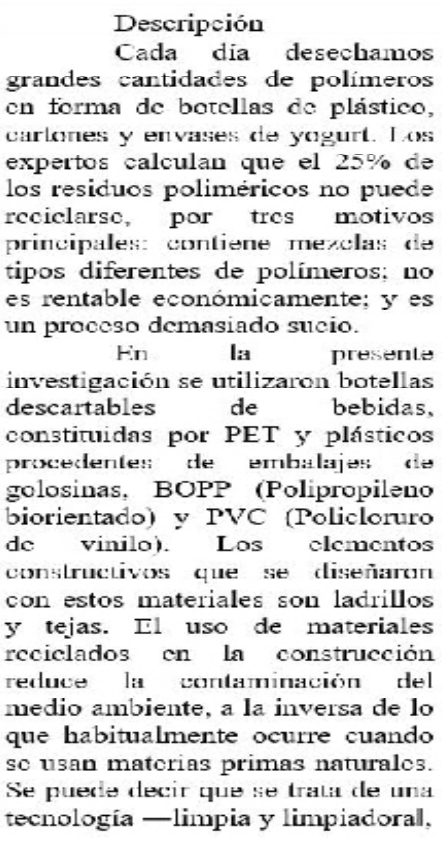

Análisis
A diario y sin darnos
cuenta todos los envases de los
cuales consumimos algún tipo do
alimento o iaquellos en losis què
algunos vienen envueltos que son
totalmente innecesarios, se tiran a
la basura se calcula que al menos
un $25 \%$ tion imposible:s de reviclar
por varios motivos: son mezclas
de varios polimeros, no es
rentable reciclarlos, o es un
procesoso aúm mát: contaminante.


Tercer análisis

\begin{tabular}{|c|c|c|}
\hline Fuente & 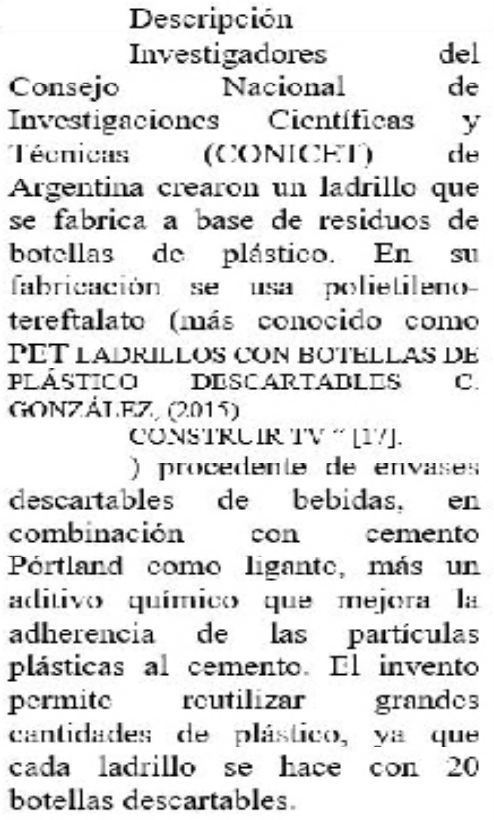 & 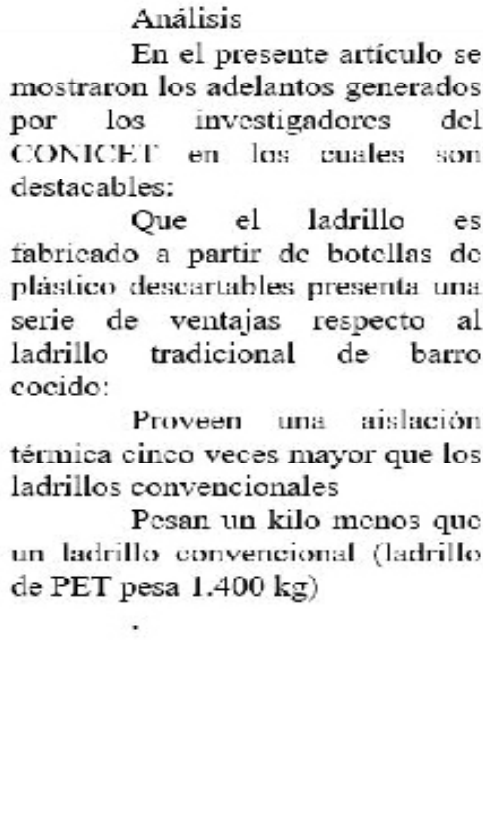 \\
\hline
\end{tabular}

\section{Discusión}

Abordando el tema del reciclaje de residuos plásticos y considerando la viabilidad del uso de Eco-ladrillos para fines de construcción, estos se forman mediante el embalaje de plástico dentro de tereftalato de polietileno (PET) en botellas. Se proporcionaron lineamientos para la construcción de estos Eco-ladrillos donde los experimentos fueron llevados a cabo para caracterizar algunas de las propiedades de estos ladrillos. Se realizaron pruebas de compresión, aislamiento acústico y demás, finamente se realizó una comparativa con el comportamiento de la construcción tradicional materiales y condiciones, además se discutieron posibles aplicaciones de los Eco-ladrillos.

Se encontró un tipo de construcción donde su materia prima se logra a través del reciclaje, utilizando para ello las botellas de desecho, donde básicamente funcionan como un sistema de mampostería, remplazando el ladrillo tradicional por botellas "ecoladrillos" y estas pueden ser de plástico PET (Polietileno Tereftalato) o de vidrio; éstas últimas pueden provocar luminosidades y efectos de colores. Este proyecto además de crear un aspecto positivo para el medio ambiente por su reutilización de basura brinda una estructura resistente, de bajo peso y garantiza las condiciones térmicas adecuadas, permitiendo dar acceso a la vivienda a familias o comunidades con bajos recursos.

Con las diferentes tomas de muestras, ensayos y caracterización de Mezclas de Tereftalato de Polietileno (PET) y Cemento. se estableció un posible comportamiento del plástico proveniente de las botellas de gaseosas al utilizarlo como agregado en una mezcla. Las mezclas de PET - Cemento realizadas están conformadas por 5\%, 10\% 


\section{2}

y $15 \%$ de PET además de arena y piedra, en proporciones y con su tipo de mezcla, es decir, si es para mortero o para concreto. Para determinar las propiedades mecánicas $y$ de durabilidad de las mezclas realizadas se elaboraron una serie de pruebas que tuvieran las características ideales.

Desde el punto de vista de resistencia y durabilidad; a compresión simple, la mezcla de concreto con un $15 \%$ de PET es la que se considera la más apropiada ya que resultó ser en promedio la más resistente aun cuando no sea la mezcla más homogénea. Por otra parte, su capacidad de absorción es baja al igual que su comportamiento ante la erosión e impacto, lo que la hace la mezcla más idónea para ser utilizada como material de construcción. Hay que destacar que no es la más costosa dentro de las tres mezclas que contienen PET.

La mezcla de PET - Cemento ayuda a reducir las cantidades de PET que no poseen una disposición final adecuada, disminuyendo así su impacto ambiental, ya que se necesita una gran cantidad de botellas de gaseosas para obtener el material para elaborar la mezcla, por lo que dichas botellas se estarían eliminando del ambiente.

\section{Conclusiones}

Para proceder a analizar ambos materiales se recolectaron todos los datos pertinentes y se encontró que contemplando la fabricación de ladrillos, el propósito de los ensayos de resistencia del concreto es la de determinar el cumplimiento de la especificación de resistencia y de medir su variabilidad, sin embargo estas variaciones también pueden ser el resultado de los procedimientos seguidos durante la dosificación, el mezclado, la colocación y el curado, además de estas, también se introducen variaciones en los ensayos por efecto de fabricación, por procedimiento de ensayo y por el tratamiento de las muestras de ensayo.

Teniendo en cuenta los parámetros establecidos en la norma NTC 2017, el porcentaje de adsorción en los ladrillos elaborados con adición de PET cumplen en todas las dosificaciones que se establecieron, ya que dicho porcentaje es menor del $12 \%$ como valor promedio establecido en dicha norma.

Teniendo como punto de comparación los ladrillos ya existentes, es posible mejorar la capacidad de carga (módulo de Rotura, (Mr.)) de los adoquines con la adición del material reciclado PET; por lo tanto, es viable el uso del PET como materia prima reciclada para la fabricación de ladrillos, ya que no se ve afectado su resistencia y por ende el desempeño de este.

El módulo de rotura (Mr.) más alto fue de 4,3 Mpa, el cual se alcanzó con las pruebas realizadas a los ladrillos con adición de PET reciclado con un $20 \%$. El porcentaje de PET más representativo en la mezcla y que cumple con todas las especificaciones es del $25 \%$, ya que cumple con la clasificación en cuanto absorción de agua y resistencia al flexo tracción (Modulo de rotura (Mr.)), esto representa una notable disminución en la arena que se requiere para su elaboración (una cuarta parte). Interpretando las gráficas, la muestra patrón fue la que obtuvo mayor resistencia a la flexión, comparada con las muestras de diferentes porcentajes de adición de fibras.

Cabe resaltar que tanto para una edad de $7 \mathrm{y}$ 28 días, la muestra que tuvo menor desviación con respecto a los ensayos realizados fue la del $20 \%$ de adición de PET. A demás, después de realizar los ensayos de Absorción y Flexión se hallaron partículas de PET que no tuvieron adherencia con la mezcla de cemento-arena, 
es decir no se observó unión ni cohesión homogénea al cemento por parte de algunas partículas en la superficie.

Después de analizar y comparar las diferentes características de ambos ladrillos se llegó a la conclusión de que Proveen una aislación térmica cinco veces mayor que los ladrillos convencionales, Pesan un kilo menos que un ladrillo convencional (ladrillo de PET pesa $1.400 \mathrm{~kg}$ ), al tener mayor aislamiento térmico, se pueden construir muros de menor espesor.

En vez de hacer paredes de $30 \mathrm{~cm}$ se pueden hacer de 15 . Esto apunta a que este ladrillo es mucho mejor en las características fundamentales para el ámbito de la construcción, y se pueden aprovechar para construir edificaciones de mayor altura, añadiendo que minimiza el uso de la energía y otros servicios básicos necesario, consiguiendo así una relación temperatura gasto energético muy optima.

\section{Referencias}

[1] J.A. Altamirano Príncipe, O.D. Bullon Westreicher, K. Cajacuri Carbonero, F.A. Chiok Meza y J. Salvatierra Arias, Ladrillo ecológicos con material reciclado Pet, trabajo de grado, Unuversidad San Ugnacio de Loyola, 2017

[2] G. Jaramillo, Manual de Materiales de construcción, Quindio: Colección Universidad del Quindio, 2019

[3] J. Toirac Corral, "El suelo-cemento como material de construcción”, Ciencia y Sociedad, vol. XXXIII, no. 4, pp. 520-571, octubrediciembre, 2008

[4] J.L. Jaya y J.L. Gomezcoello, “Análisis comparativo de la contaminación atmosférica producida por la combustión en ladrilleras artesanales utilizando tres tipos de combustibles", trabajo de grado, Universidad Politécnica Salesiana Sede Cuenca, 2012

[5] M. Sánchez Silva, L.A. Zapata Valencia, "Impacto ambiental y gestión del riesgo de ladrilleras en la Vereda Los Gómez de Itagui”, Cuaderno Activa, no. 5, pp. 109-123, 2013

[6] V. Flores, J. Rojas, R. Torres, R Vallejos, P. Flores y M. Flores, Mezclas de cemento y agregados de plástico para la construcción de viviendas ecológicas, Ciencias Tecnológicas y Agrarias T-I: Handbook, 2014

[7] A. Alfonzo Ortega, Eco-ladrillo a base de caucho reciclado de neumáticos fuera de uso, trabajo de grado, Universidad Nacional de Asunción, 2016

[8] S. Barno, "Ladrillos Pet" a base de residuos plásticos, sostenibilidad y arquitectura., 2016. [En línea]. Disponible en: https://www. sostenibilidadyarquitectura.com/2016/09/09/ ladrillos-pet-a-base-de-residuos-plasticos/

[9] C. Paredes, Comparativa distintos tipos de muros: nuevos y tradicionales, 2015. [En línea]. Disponible en: http://www.paredestudio. com.ar/ventajas-desventajas-constructivos/\#. XQfTX4hKhPY

[10] R. Gaggino, Botellas descartables para casas sustentables, Consejo Nacional de Investigaciones, 2015. [En línea]. Disponible en:https://www.conicet.gov.ar/botellasdescartables-para-casas-sustentables/

[11] P. Garcidueñas, Conoce los ladrillos hechos de plástico reciclado. Expok Comunicación de sustentabilidad y RSE, 2016. [En línea]. Disponible en: https://www.expoknews.com/ conoce-los-ladrillos-hechos-de-plasticoreciclado/ 


\section{4}

[12] A. Martínez Amariz y M. Cote Jiménez, Diseño y fabricación de ladrillo reutilizando materiales a base de PET, INGE CUC, vol. 10, no. 2 , pp. 76 - 80, dic. 2014

[13] F. López-Noguero, "El Análisis de contenido como método de investigación", Revista de educación, no. 4, pp. 167-180, 2002

[14] P.J. Angumba Aguilar, "Ladrillos elaborados con plástico reciclado (PET) para mampostería no portante", trabajo de grado, Universidad de Cuenca, 2018 